\title{
Birkhoff Center of an Almost Distributive Fuzzy Lattice
}

\author{
Berhanu Assaye Alaba and Gerima Tefera Dejen
}

\begin{abstract}
The concept of Birkhoff center $B_{A}(R)$ of an Almost distributive fuzzy lattice $(R, A)$ with maximal element is introduced. We also prove that $B_{A}(R)$ is relatively complemented ADFL and product of ADFL is a gain ADFL.

Index Terms-Almost distributive fuzzy lattice, almost distributive lattice, Birkhoff center of an almost distributive fuzzy lattice, Birkhoff center of an almost distributive lattice, fuzzy poset, relatively complemented ADFL.
\end{abstract}

\section{INTRODUCTION}

$\mathbf{T}$ HE concept of an Almost distributive lattice was introduced by U.M. Swamy and G.C. Rao in [1]. In [2], U.M. Swamy, G.C. Rao, R.V.G. Ravi Kummar and Ch. Pragathi have extended the above concept for a general partial ordered set $P$ and prove that $B(P)$ is relatively complemented distributive lattice in which the operations are least upper bound and greatest lower bound in $P$ (provided that $B(P)$ is non-empty in Birkhoff center of an ADL).

The concept of Birkhoff center $B(R)$ of an ADL with maximal elements was introduced by U.M. Swamy and S. Ramesh in [3] and prove that $B(R)$ is a relatively complemented Almost distributive lattice. The concept of a fuzzy set was first introduced by Zadeh in [4], and this concept was adapted by Goguen in [5] and Sanchez in [6] to define and study fuzzy relations. Yuan and $\mathrm{Wu}$ in [7] introduced the concepts of fuzzy lattices and fuzzy ideal of a lattice. As a continuation of these studies, we define fuzzy relation, fuzzy poset and fuzzy lattice which enables us to define Birkhoff center of Almost distributive fuzzy lattice. In this paper, we introduce the concept of the Birkhoff center $B_{A}(R)$ of an Almost distriutive fuzzy lattice (ADFL) with maximal elements and prove that $B_{A}(R)$ is a relatively complemented Almost distributive fuzzy lattice. Mainly we obtain the equivalency of the Birkhoff center in ADL to the the Birkhoff center of ADFL with the property of Almost distributive lattice and Fuzzy partial order relation. Throughout this paper we consider only $A D F L_{S}$ which contain at least one maximal element. $(R, A)$ denotes an ADFL. An $\operatorname{ADL}(R, \vee, \wedge, 0)$ represented by $R$ and $x \in(R, A) \Leftrightarrow x \in R$.

\section{PRELIMINARIES}

Definition 1 ([8]): An algebra $(R, \vee, \wedge, 0)$ of type $(2,2,0)$ is said to be an Almost distributive lattice(ADL) if it satisfies the

Manuscript received April 29, 2017; accepted June 21, 2017. This work was supported by the Ethiopian Ministry of Education under the sponsorship of Bahir Dar University and Wollo University.

The authors are with the Department of Mathematics, College of Science, Bahir Dar University, Bahir Dar, Ethiopia. Email: berhanu_assaye@yahoo.com, gerima233@gmail.com following conditions:

(1) $a \vee 0=a$.

(2) $0 \wedge a=0$.

(3) $(a \vee b) \wedge c=(a \wedge c) \vee(b \wedge c)$.

(4) $a \wedge(b \vee c)=(a \wedge b) \vee(a \wedge c)$

(5) $a \vee(b \wedge c)=(a \vee b) \wedge(a \vee c)$

(6) $(a \vee b) \wedge b=b, \quad \forall a, b, c \in R$.

The element 0 is called as usual the zero element of $\mathrm{R}$.

Definition 2 ([8]): Let $\mathrm{X}$ be a non-empty set. Fix $x_{o} \in X$. For any $x, y \in X$,

$$
x \wedge y= \begin{cases}x_{o}, & \text { if } x=x_{o} \\ y, & \text { if } x \neq x_{o}\end{cases}
$$

and

$$
x \vee y= \begin{cases}y, & \text { if } x=x_{o} \\ x, & \text { if } x \neq x_{o}\end{cases}
$$

Then $\left(X, \vee, \wedge, x_{o}\right)$ is an ADL with $x_{o}$ as its zero element. This ADL is called a discrete ADL.

Example 3: Every distributive lattice with zero is an ADL. For any $a, b$ in an ADL $R$, we say that $a$ is less than or equal to $b$ and write $a \leq b$, if $a \wedge b=a$. Then $\leq$ is a partial ordering on $R$.

Lemma 4 ([8]): For any $a, b \in R$, we have

(1) $a \wedge 0=0$ and $0 \vee a=a$.

(2) $a \wedge a=a=a \vee a$.

(3) $(a \wedge b) \vee b=b, a \vee(b \wedge a)=a$ and $a \wedge(a \vee b)=a$.

(4) $a \wedge b=b \Leftrightarrow a \vee b=a$.

(5) $a \wedge b=a \Leftrightarrow a \vee b=b$.

(6) $a \wedge b \leq b$ and $a \leq a \vee b$.

(7) $a \wedge b=b \wedge a$, whenever $a \leq b$.

(8) $a \vee(b \vee a)=a \vee b$.

Theorem 5 ([8]): For any $a, b \in R$, the following are equivalent to each other:

(1) $(a \wedge b) \vee a=a$.

(2) $a \wedge(b \vee a)=a$.

(3) $(b \wedge a) \vee b=b$.

(4) $b \wedge(a \vee b)=b$.

(5) $a \wedge b=b \wedge a$.

(6) $a \vee b=b \vee a$

(7) The supremum of $a$ and $b$ exists and equal to $a \vee b$.

(8) There exists $x \in R$ such that $a \leq x$ and $b \leq x$.

(9) The infimum of $a$ and $b$ exists and equal to $a \wedge b$.

Theorem 6 ([8]): For any $a, b \in R$ we have

(1) $(a \vee b) \wedge c=(b \vee a) \wedge c$.

(2) $\wedge$ is associative in $R$.

(3) $a \wedge b \wedge c=b \wedge a \wedge c$. 
From the above theorem, it follows that for any $x \in R$ the set $\{a \wedge x \mid a \in R\}$ forms a bounded distributive lattice. In particular, we have $((a \wedge b) \vee c) \wedge x=((a \vee c) \wedge(b \vee c)) \wedge x$, for all $a, b, c, x \in R$.

An element $m \in R$ is said to be maximal if $m \leq x$ implies $m=x$.

Lemma 7: Let $R$ be an ADL with 0 , and $m \in R$. Then the following are equivalent:

(1) $m$ is a maximal element with respect to the partial ordering " $\leq . "$

(2) $m \vee x=m$, for all $x \in R$.

(3) $m \wedge x=x$, for all $x \in R$.

Definition 8 ([8]): A non-empty subset $I$ of $R$ is said to be an ideal of $R$ if it satisfies the following conditions:

(1) $a, b \in I \Rightarrow a \vee b \in I$.

(2) $a \in I, x \in R \Rightarrow a \wedge x \in I$.

Theorem 9 ([8]): The following are equivalent for any ADL $R$ :

(1) $R$ is relatively complemented.

(2) Given $x, y \in R$, there exists $a \in R$ such that $x \wedge a=0$ and $x \vee a=x \vee y$.

(3) For any $x \in R$, the interval $[0, x]$ is complemented.

Theorem 10 ([8]): A relatively complemented ADL $R$ is associative.

Definition 11 ([8]): An $\operatorname{ADL}(R, \vee, \wedge)$ is said to be relatively complemented if every interval $[a, b], a \leq b$ in $R$ is a complemented lattice.

Definition 12 ([9]): Let $X$ be a non- empty set, a function $A: X \times X \rightarrow[0,1]$ is said to be fuzzy partial order relation if it satisfies the following conditions:

(1) $A(x, x)=1, \forall x \in X$ that is $\mathrm{A}$ is reflexive.

(2) $A(x, y)>0$, and $A(y, x)>0$ implies that $x=y$. That is $\mathrm{A}$ is antisymmetric.

(3) $A(x, z) \geq \sup _{y \in X} \min [A(x, y), A(y, z)]>0$. That is $\mathrm{A}$ is transitive.

If $A$ is a fuzzy partial order relation in a set $X$, then $(X, A)$ is called a fuzzy partial order relation or fuzzy poset.

Definition 13 ([9]): Let $(X, A)$ be a fuzzy poset. Then $(X, A)$ is a fuzzy lattice if and only if $x \vee y$, and $x \wedge y$ exists for all $x, y \in X$.

Definition 14 ([9]): Let $(X, A)$ be a fuzzy lattice.Then $(X, A)$ is distributive if and only if $x \wedge(y \vee z)=(x \wedge y) \vee(x \wedge z)$, and $(x \vee y) \wedge(x \vee z)=x \vee(y \wedge z)$, forall, $x, y, z \in X$.

Definition 15 ([3]): Given an with maximal element. Define $B(R)=$ $\{a \in R \mid a \wedge b=0$, and $a \vee b$ is maximal for some $b \in R\}$.

Then $B(R)$ is called the Brikhoff center of R. Let $a \wedge b=0$, $a \vee b$ is maximal .Then $b \wedge a=0$, and $b \vee a$ is maximal, in this case $a$ and $b$ are called complements to each other.

Theorem 16: For any $a \in R, a \in B(R)$ if and only if there exist two sub $A D L_{s} R_{1}$, and $R_{2}$ of $R$ with maximal elements and an isomorphism $f: R \rightarrow R_{1} \times R_{2}$ such that $f(a)=\left(m_{1}, 0\right)$, where $m_{1}$ is a maximal element in $R_{1}$.

Definition 17 ([10]): Let $(R, \vee, \wedge, 0)$ be an algebra of type $(2,2,0)$ and we call $(R, A)$ is an Almost Distributive Fuzzy Lattice(ADFL) if the following conditions satisfied:

$\left(F_{1}\right) A(a, a \vee 0)=A(a \vee 0, a)=1$.

$\left(F_{2}\right) A(0,0 \wedge a)=A(0 \wedge a, 0)=1$.
$\left(F_{3}\right) A((a \vee b) \wedge c,(a \wedge c) \vee(b \wedge c))=A((a \wedge c) \vee(b \wedge c),(a \vee$ b) $\wedge c)=1$.

$\left(F_{4}\right) A(a \wedge(b \vee c),(a \wedge b) \vee(a \wedge c))=A((a \wedge b) \vee(a \wedge c), a \wedge$ $(b \vee c))=1$.

$\left(F_{5}\right) A(a \vee(b \wedge c),(a \vee b) \wedge(a \vee c))=A((a \vee b) \wedge(a \vee c), a \vee$ $(b \wedge c))=1$.

$\left(F_{6}\right) A((a \vee b) \wedge b, b)=A(b,(a \vee b) \wedge b)=1$, for all $a, b, c \in R$.

\section{BIRKHOFF CENTER OF Almost Distributive FuZZY} LATTICE

Definition 18: Let $(R, A)$ be an ADFL. Then an element $m$ of $(R, A)$ is a maximal element of $(R, A)$ if, for any $x \in R$, $A(m, x)>0$ implies $A(x, m)>0$.

Proposition 19: Let $(R, A)$ be an ADFL.Then an element $\mathrm{m}$ of $\mathrm{R}$ is a maximal element of $(R, A)$ if and only if $A(m \vee x, m)>$ 0 ,for all $x \in R$.

Proof: Assume $m \in R$ be a maximal element.Then there exist $x \in R$ such that $m \leq m \vee x$, for all $x \in R$. Then $A(m, m \vee$ $x)>0$. Now, $A(m \vee x, m)=A(m \vee(m \wedge x), m)=A(m, m)=1>$ 0 , for all $x \in R$. Hence $A(m \vee x, m)>0$. Conversely, Suppose $A(m \vee x, m)>0$, for all $x \in R$, and $m \in R$, Since $m \leq m \vee x$. So that we get $A(m, m \vee x)>0$. Hence $m \vee x=m$ by anti symmetric property of A. Therefore, $m$ is maximal element in $R$.

Definition 20: Let $(R, A)$ be an ADFL with maximal element. Define $B_{A}(R)=\{a \in R \mid A(a \wedge b, 0)>0$ and $A((a \vee b) \vee$ $x, a \vee b)>0$ for some $b \in R$, for all $x \in R\}$. Then $B_{A}(R)$ is called the Birkhoff center of $(R, A)$.

Lemma 21: If $A(a \wedge b, 0)>0$, and $A((a \vee b) \vee x, a \vee b)>0$, for all $x \in R$, then $A(b \wedge a, 0)>0$ and $A((b \vee a) \vee x, b \vee a)>0$, for all $x \in R$.

Proof: Assume the first condition holds. Let $a \in B_{A}(R)$. Then there exist $b \in R$ and $A(b \wedge a, 0)$

$=A((b \wedge a) \wedge(a \vee b), 0)$

$=A([(b \wedge a) \wedge a] \vee[(b \wedge a) \wedge b], 0)$

$=A((b \wedge a) \vee(a \wedge(b \wedge b), 0)$, since $b \wedge b=b$, and $b \wedge a \wedge b=a \wedge b \wedge b=$

$A([(b \wedge a) \vee a] \wedge[(b \wedge a) \vee b], 0)$

$=A(a \wedge[(b \wedge a) \vee b], 0)$

$=A([a \wedge(b \wedge a)] \vee(a \wedge b), 0)$

$=A([(a \wedge b) \wedge a] \vee o, 0)$

$=A([0 \wedge a] \vee 0,0)$

$=A(0,0)=1>0$.

Hence $A(b \wedge a, 0)>0$.

Assume $A((a \vee b) \vee x, a \vee b)>0$, for all $x \in R$

$A((b \vee a) \vee x, b \vee a)=A([b \vee(a \wedge(a \vee b)] \vee x,[b \vee(a \wedge(a \vee b)])$

$=A([(b \vee a) \wedge(b \vee(a \vee b))] \vee x,(b \vee a) \wedge(b \vee(a \vee b)))$

$=A([(b \vee a) \wedge(a \vee b)] \vee x,(b \vee a) \wedge(a \vee b))$

$=A([((b \vee a) \wedge a) \vee((b \vee a) \wedge b)] \vee x,((b \vee a) \wedge a) \vee((b \vee a) \wedge$

$b))$ by Lemma 4 and Theorem 5 and Proposition 19

$=A([a \vee b] \vee x, a \vee b)>0$

Hence $A((b \vee a) \vee x, b \vee a)>0$ for all $x \in R$.

Example 22: Let $X=\{\{0, a\} \mid a \in X\}$ with zero element 0 and $a \neq 0$. Define $\vee$ and $\wedge$ by the following table.

\begin{tabular}{|c|c|c|}
\hline$\vee$ & 0 & $\mathrm{a}$ \\
\hline 0 & 0 & $\mathrm{a}$ \\
\hline $\mathrm{a}$ & $\mathrm{a}$ & $\mathrm{a}$ \\
\hline
\end{tabular} and \begin{tabular}{|c|c|c|}
\hline$\wedge$ & 0 & $\mathrm{a}$ \\
\hline 0 & 0 & 0 \\
\hline $\mathrm{a}$ & 0 & $\mathrm{a}$ \\
\hline
\end{tabular}


then $(X, \vee, \wedge, 0)$ is an ADL which is a discrete ADL. Since every non-zero element is maximal. $a$ is maximal element. $0 \wedge a=a \wedge 0=0$ and $a=a \vee 0$ is maximal element of $\mathrm{X}$. Implies that $a \in B(X)$. Let $A: X \times X \rightarrow[0,1]$ be a fuzzy partial order relation defined by $A(0,0)=A(a, a)=1$ and $A(0, a)=0$, $A(a, 0)=0.3$. Then $(X, A)$ is a fuzzy poset. $A(0,0 \wedge a)=A(0 \wedge$ $a, 0)=1$, and $A((0 \vee a) \vee a, 0 \vee a)=A(a \vee a, a)=A(a, a)=1>$ 0 , since $0 \vee a=a$, and $a \vee a=a$. Hence $a \in B_{A}(R)$.

Definition 23: Let $(R, A)$ be an ADFL and $R_{1}$ be an ideal of R.Then $\left(R_{1}, A_{1}\right)$ is an ideal of $(R, A)$.

(1) If $a \in R, b \in R_{1}$ and $A_{1}(a, b)>0$, then $a \in\left(R_{1}, A_{1}\right)$.

(2) If $a, b \in R_{1}$, then $a \vee b \in\left(R_{1}, A_{1}\right)$

Definition 24: Let $(R, A)$ be an ADFL. Then a non-empty subset $\mathrm{K}$ of an ADFL is said to be a sub- ADFL of $(R, A)$ if $K$ is closed under induced operation of $(R, A)$.

Lemma 25: Let $(R, A)$ be an ADFL and $R_{1}$ be an ideal of R.Then $\left(R_{1}, A_{1}\right)$ is a sub- ADFL of $(R, A)$.

Proof: Let $\left(R_{1}, A_{1}\right)$ be an ideal of $(R, A)$, where $R_{1}$ is an ideal of R.Then

(1) If $a \in R, b \in R_{1}$ and $A_{1}(a, b)>0$, then $a \in R_{1}$ by definition of ideal of $(R, A)$. Again if $a \in R, c \in R_{1}$ and $A_{1}(a, c)>0$, then $a \in R_{1}$. Now, $A_{1}(a, b)>0$ and $A_{1}(a, c)>0 \Rightarrow A_{1}(a, b \wedge c)>0$. $\Rightarrow A_{1}(b \wedge c, c)>0 b \wedge c \in R_{1} . \Rightarrow b \wedge c \in\left(R_{1}, A_{1}\right)$.

(2) If $a, b \in R_{1}$, then $a \vee b \in R_{1} . \Rightarrow a \vee b \in\left(R_{1}, A_{1}\right)$. Hence $\left(R_{1}, A_{1}\right)$ is a sub- ADFL of $(R, A)$.

Definition 26: Let $\left(R_{1}, A_{1}\right)$ and $\left(R_{2}, A_{2}\right)$ be two $A D F L_{s}$. Then the following point wise operations holds for any $(a, c) \in$ $R_{1} \times R_{2}$ and $(b, d) \in R_{1} \times R_{2}$.

(1) $\left(A_{1} \times A_{2}\right)((a, c) \wedge(b, d),(a \wedge b, c \wedge d))=\left(A_{1} \times A_{2}((a \wedge b, c \wedge\right.$ d), $(a, c) \wedge(b, d))=1$.

(2) $\left(A_{1} \times A_{2}\right)((a, c) \vee(b, d),(a \vee b, c \vee d))=\left(A_{1} \times A_{2}((a \vee b, c \vee\right.$ d), $(a, c) \vee(b, d))=1$.

Definition 27: Let $\left(R_{1}, A_{1}\right)$ and $\left(R_{2}, A_{2}\right)$ be two $A D F L_{s}$. Then $\left(A_{1} \times A_{2}\right)((a, b),(c, d))=\min \left\{A_{1}(a, c), A_{2}(b, d)\right\}$.

Definition 28: Let $\left(R_{1}, A_{1}\right)$ and $\left(R_{2}, A_{2}\right)$ be two $A D F L_{S}$. Then a mapping $f:\left(R_{1}, A_{1}\right) \rightarrow\left(R_{2}, A_{2}\right)$ is said to be a fuzzy latice homomorphism. If it satisfy the following condition for any $x, y, 0 \in R_{1}$ :

(1) $A_{2}(f(x \wedge y), f(x) \wedge f(y))=A_{2}(f(x) \wedge f(y), f(x \wedge y))=1$.

(2) $A_{2}(f(x \vee y), f(x) \vee f(y))=A_{2}(f(x) \vee f(y), f(x \vee y))=1$.

(3) $A_{2}(f(0), 0)>0$.

Lemma 29: Let $\left(R_{1}, A_{1}\right)$ and $\left(R_{2}, A_{2}\right)$ be two $A D F L_{S}$, and $f:\left(R_{1}, A_{1}\right) \rightarrow\left(R_{2}, A_{2}\right)$ be any mapping. Then $f(x)=f(y) \Leftrightarrow$ $A_{2}(f(x), f(y))=A_{2}(f(y), f(x))=1$, for any $x, y \in R_{1}$.

Proof: Assume $f(x)=f(y)$,for all $x, y \in R_{1}$. Now $A_{2}(f(x), f(y))=A_{2}(f(x), f(x))=1, \quad$ since $f(x)=f(y)$. Similarly $A_{2}(f(y), f(x))=1$. Hence $A_{2}(f(x), f(y))=$ $A_{2}(f(y), f(x))=1$. Conversely, suppose $A_{2}(f(x), f(y))=$ $A_{2}(f(y), f(x))=1$, for all $x, y \in R_{1}$

$\Rightarrow A_{2}(f(x), f(y))=1>0$

$\Rightarrow A_{2}(f(x), f(y))>0$.

Similarly $A_{2}(f(y), f(x))>0$. Therefore $f(x)=$ $f(y)$, for any $x, y \in R_{1}$, by anti symmetry property of $A_{2}$.

Definition 30: If $\left(R_{1}, A_{1}\right)$, and $\left(R_{2}, A_{2}\right)$ are $A D F L_{s}$ with maximal elements $m_{1}$ and $m_{2}$ respectively. Then $\left(R_{1} \times\right.$ $\left.R_{2}, A_{1} \times A_{2}\right)$ has maximal element $\left(m_{1}, m_{2}\right)$ if and only if $A_{1}\left(m_{1}, x\right)>0 \Rightarrow A_{1}\left(x, m_{1}\right)>0$ and $A_{2}\left(m_{2}, y\right)>0 \Rightarrow$ $A_{2}\left(y, m_{2}\right)>0$ for all $x \in R_{1}$ and for all $y \in R_{2}$.

Lemma 31: Let $\left(R_{1}, A_{1}\right)$ and $\left(R_{2}, A_{2}\right)$ be two $A D F L_{s}$. Then $\left(R_{1} \times R_{2}, A_{1} \times A_{2}\right)$ is an ADFL.

Proof: Let $(a, b) \in R_{1} \times R_{2}$ and $(0,0) \in R_{1} \times R_{2}$.

(1) $\left(A_{1} \times A_{2}\right)((a, b) \vee(0,0),(a, b))=\left(A_{1} \times A_{2}\right)((a \vee 0, b \vee$ $0),(a, b))$

$=\min \left\{A_{1}(a \vee 0, a), A_{2}(b \vee 0, b)\right\}=\min \left\{A_{1}(a, a), A_{2}(b, b)\right\}$ $=\min \{1,1\}=1$

Hence $\left(A_{1} \times A_{2}\right)((a, b) \vee(0,0),(a, b))=1$. Similarly $\left(A_{1} \times\right.$ $\left.A_{2}\right)((a, b),(a, b) \vee(0,0))=1$. We have $\left(A_{1} \times A_{2}\right)((a, b) \vee$ $(0,0),(a, b))=\left(A_{1} \times A_{2}\right)((a, b),(a, b) \vee(0,0))=1$.

(2) $\left(A_{1} \times A_{2}\right)((0,0),(0,0) \wedge(a, b))=\left(A_{1} \times A_{2}((0,0),(0 \wedge a, 0 \wedge\right.$ b))

$=\min \left\{A_{1}(0,0 \wedge a), A_{2}(0,0 \wedge b)\right\}=\min \left\{A_{1}(0,0), A_{2}(0,0)\right\}$

$=\min \{1,1\}=1$.

Hence $\left(A_{1} \times A_{2}\right)((0,0),(0,0) \wedge(a, b))=1$.

Similarly $\left(A_{1} \times A_{2}\right)((a, b) \wedge(0,0),(0,0))=1$. Therefore $\left(A_{1} \times\right.$ $\left.A_{2}\right)((0,0),(0,0) \wedge(a, b))=\left(A_{1} \times A_{2}\right)((a, b) \wedge(0,0),(0,0))=1$.

(3) Let $a, b, c \in R_{1}$ and $d, e, h \in R_{2}$. Then $\left(A_{1} \times A_{2}\right)((a \wedge(b \vee$ $c), d \wedge(e \vee h)),((a \wedge b) \vee(a \wedge c),(d \wedge e) \vee(d \wedge h)))$

$=\min \left\{A_{1}(a \wedge(b \vee c),(a \wedge b) \vee(a \wedge c))\right.$,

$\left.\left.A_{2}(d \wedge(e \vee h),(d \wedge e) \vee(d \wedge h))\right)\right\}$

$=\min \left\{A_{1}(a \wedge(b \vee c), a \wedge(b \vee c))\right.$,

$\left.A_{2}(d \wedge(e \vee h), d \wedge(e \vee h))\right\}$ by $L D \wedge$.

$=\min \{1,1\}=1$.

Hence $\left(A_{1} \times A_{2}\right)((a \wedge(b \vee c), d \wedge(e \vee h)),((a \wedge b) \vee(a \wedge c),(d \wedge$ $e) \vee(d \wedge h)))=1$. Similarly $\left(A_{1} \times A_{2}\right)((((a \wedge b) \vee(a \wedge c),(d \wedge$ $e) \vee(d \wedge h)),(a \wedge(b \vee c), d \wedge(e \vee h)))=1$. We have $\left(A_{1} \times\right.$ $\left.A_{2}\right)((a \wedge(b \vee c), d \wedge(e \vee h)),((a \wedge b) \vee(a \wedge c),(d \wedge e) \vee(d \wedge$ $h)))=\left(A_{1} \times A_{2}\right)(((a \wedge b) \vee(a \wedge c),(d \wedge e) \vee(d \wedge h)),(a \wedge(b \vee$ $c), d \wedge(e \vee h)))=1$. In the same manner the remaining property holds. Thus $\left(R_{1} \times R_{2}, A_{1} \times A_{2}\right)$ is an ADFL.

Theorem 32: For any $x \in R, x \in B_{A}(R)$ if and only if there exist two sub $-A D F L_{S},\left(R_{1}, A_{1}\right)$, and $\left(R_{2}, A_{2}\right)$ with maximal elements and an isomorphism $f:(R, A) \rightarrow\left(R_{1} \times R_{2}, A_{1} \times A_{2}\right)$ such that $\left(A_{1} \times A_{2}\right)(f(x),(a, 0))=\left(A_{1} \times A_{2}\right)((a, 0), f(x))=1$, where $a$ is the maximal element of $\left(R_{1}, A_{1}\right)$.

Proof: Let $(R, A)$ be an ADFL. Suppose $a \in B_{A}(R)$ and $a \in R$.Then there exist $d, b \in R$ such that $\left(A_{1} \times A_{2}\right)((a \wedge b, a \wedge d),(0,0))>0$ and $\left.\left(A_{1} \times A_{2}\right)((a \vee b) \vee x,(a \vee d) \vee y),(a \vee b, a \vee d)\right)>0$. Define $f:(R, A) \rightarrow\left(R_{1} \times R_{2}, A_{1} \times A_{2}\right)$ by $\left(A_{1} \times\right.$ $\left.A_{2}\right)(f(x),(a \wedge x, b \wedge x))=\left(A_{1} \times A_{2}\right)((a \wedge x, b \wedge x), f(x))=1$. Let $x, y \in R$. Then $\left(A_{1} \times A_{2}\right)(f(x \wedge y), f(x) \wedge f(y))=$ $\left(A_{1} \times A_{2}\right)((a \wedge(x \wedge y), b \wedge(x \wedge y)),((a \wedge x, b \wedge x) \wedge(a \wedge y, b \wedge y))$ $=\min \left\{A_{1}(a \wedge(x \wedge y),(a \wedge x) \wedge(a \wedge y)), A_{2}(b \wedge(x \wedge y)\right.$,

$(b \wedge x) \wedge(b \wedge y))\}$

$=\min \left\{A_{1}(a \wedge(x \wedge y), a \wedge(x \wedge y)), A_{2}(b \wedge(x \wedge y), b \wedge(x \wedge y))\right\}$

by $L D \wedge$.

$=\min \{1,1\}=1$.

Hence $\left(A_{1} \times A_{2}\right)(f(x \wedge y), f(x) \wedge f(y))=1$. Similarly $\left(A_{1} \times A_{2}\right)(f(x) \wedge f(y), f(x \wedge y))=1$. We have $\left(A_{1} \times A_{2}\right)(f(x \wedge$ $y), f(x) \wedge f(y))=\left(A_{1} \times A_{2}\right)(f(x) \wedge f(y), f(x \wedge y))=1$. $\left(A_{1} \times A_{2}\right)(f(x \vee y), f(x) \vee f(y))$

$=\left(A_{1} \times A_{2}\right)((a \wedge(x \vee y), b \wedge(x \vee y)),(a \wedge x, b \wedge x) \vee(a \wedge y, b \wedge y))$ $=\left(A_{1} \times A_{2}\right)((a \wedge(x \vee y), b \wedge(x \vee y)),((a \wedge x) \vee(a \wedge y),(b \wedge x) \vee$ $(b \wedge y))$ 
$=\min \left\{A_{1}(a \wedge(x \vee y),(a \wedge x) \vee(b \wedge y))\right.$,

$\left.A_{2}(b \wedge(x \vee y),(b \wedge x) \vee(b \wedge y))\right\}$

$=\min \left\{A_{1}(a \wedge(x \vee y), a \wedge(x \vee y)), A_{2}(b \wedge(x \vee y), b \wedge(x \vee y))\right\}$

$=\min \{1,1\}=1$.

Hence $\left(A_{1} \times A_{2}\right)(f(x \vee y), f(x) \vee f(y))=1$. Similarly, $\left(A_{1} \times A_{2}\right)(f(x) \vee f(y), f(x \vee y))=1$. Hence $\left(A_{1} \times A_{2}\right)(f(x \vee$ $y), f(x) \vee f(y))=\left(A_{1} \times A_{2}\right)(f(x) \vee f(y), f(x \vee y))=1$. $\left(A_{1} \times A_{2}\right)(f(0),(0,0))=\left(A_{1} \times A_{2}\right)((a \wedge 0, b \wedge 0),(0,0))$, since $f(0)=(a \wedge 0, b \wedge 0)$.

$=\min \left\{A_{1}(a \wedge 0,0), A_{2}(b \wedge 0,0)\right\}=\min \left\{A_{1}(0,0), A_{2}(0,0)\right\}$, since $a \wedge 0=0, b \wedge 0=0$.

$=\min \{1,1\}=1>0$.

Hence $\left(A_{1} \times A_{2}\right)(f(0),(0,0))>0$. Thus, $f$ is a fuzzy lattice homomorphism. Let $x, y \in R$ and $\left(A_{1} \times A_{2}\right)(f(x), f(y))=\left(A_{1} \times A_{2}\right)(f(y), f(x))=1$. Then, $\left(A_{1} \times\right.$ $\left.A_{2}\right)(x, y)=\left(A_{1} \times A_{2}\right)((a \vee b) \wedge x, y)$, since $a \vee b$ is maximal

$=\left(A_{1} \times A_{2}\right)((a \wedge x) \vee(b \wedge x), y)$

$=\left(A_{1} \times A_{2}\right)((a \wedge y) \vee(b \wedge y), y)$, replace $x$ by $y$

$=\left(A_{1} \times A_{2}\right)((a \vee b) \wedge y, y)$

$=\left(A_{1} \times A_{2}\right)(y, y)=1>0$, since $a \vee b$ is maximal, so that we get $\left(A_{1} \times A_{2}\right)(x, y)>0$. Similarly, $\left(A_{1} \times A_{2}\right)(y, x)>0$. Hence $x=y$ by antisymmetry property of $A_{1} \times A_{2}$. Therefore $f$ is monomorphism. Let $(R, A)$ be an ADFL. Suppose $(a \wedge x, b \wedge y) \in R_{1} \times R_{2}$. Write $w=(a \wedge x) \vee(b \wedge y)$. Now, $\left(A_{1} \times A_{2}\right)(f(w),(a \wedge x, b \wedge y))$

$=\left(A_{1} \times A_{2}\right)((a \wedge w, b \wedge w),(a \wedge x, b \wedge y))$

$=\left(A_{1} \times A_{2}\right)(((a \wedge[(a \wedge x) \vee(b \wedge y)], b \wedge[(a \wedge x) \vee(b \wedge y)]),(a \wedge$ $x, b \wedge y))$

$=\left(A_{1} \times A_{2}\right)(((a \wedge(a \wedge x)) \vee(a \wedge(b \wedge y)),(b \wedge(a \wedge x)) \vee(b \wedge$ $(b \wedge y))),(a \wedge x, b \wedge y))$

$=\left(A_{1} \times A_{2}\right)(((a \wedge x) \vee((a \wedge b) \wedge y)),((b \wedge a) \wedge x)) \vee((b \wedge b) \wedge$ $y))),(a \wedge x, b \wedge y))$

$=\left(A_{1} \times A_{2}\right)(((a \wedge x) \vee 0,0 \vee(b \wedge y)),(a \wedge x, b \wedge y))$, since $a \wedge b=0$ and $b \wedge a=0$.

$=\left(A_{1} \times A_{2}\right)((a \wedge x, b \wedge y),(a \wedge x, b \wedge y))$

$=\min \left\{A_{1}(a \wedge x, a \wedge x), A_{2}(b \wedge y, b \wedge y)\right\}=\min \{1,1\}=1$.

Hence $\quad\left(A_{1} \times A_{2}\right)(f(w),(a \wedge x, b \wedge y))>0$. Similarly, $\quad\left(A_{1} \times A_{2}\right)((a \wedge x, b \wedge y), f(w))>0$. Hence $f(w)=(a \wedge x, b \wedge y) \Leftrightarrow\left(A_{1} \times A_{2}\right)(f(w),(a \wedge x, b \wedge y))=$ $\left(A_{1} \times A_{2}\right)((a \wedge x, b \wedge y), f(w))=1$ by antisymmetric property of $A_{1} \times A_{2}$. Hence $f$ is epimorphism. Therefore, $f$ is an isomorphism. Conversely, Suppose the map $f:(R, A) \rightarrow\left(R_{1} \times R_{2}, A_{1} \times A_{2}\right)$ be an isomorphism defined by $\left(A_{1} \times A_{2}\right)(f(x),(a, 0))=\left(A_{1} \times A_{2}\right)((a, 0), f(x))=1, x \in R$, where $a$ is the maximal element of $\left(R_{1}, A_{1}\right)$. Choose a maximal element $b$ in $\left(R_{2}, A_{2}\right)$ such that $\left(A_{1} \times A_{2}\right)(f(y),(0, b))=\left(A_{1} \times A_{2}\right)((0, b), f(y))=1$ for $y \in R$. Now, $\left(A_{1} \times A_{2}\right)(f(x \wedge y),(0,0))=\left(A_{1} \times A_{2}\right)(f(x) \wedge f(y),(0,0))$ $=\left(A_{1} \times A_{2}\right)((a, 0) \wedge(0, b),(0,0))=\left(A_{1} \times A_{2}\right)(a \wedge 0,0 \wedge$ b), $(0,0))$

$=\min \left\{A_{1}(0,0), A_{2}(0,0)\right\}=\min \{1,1\}=$ since $a \wedge 0=0,0 \wedge b=0$

$=\left(A_{1} \times A_{2}\right)(f(x \wedge y), f(0))>0$, since $f(0)=(0,0)$.

Similarly, $\left(A_{1} \times A_{2}\right)(f(0), f(x \wedge y))>0$

$\Rightarrow f(x \wedge y)=f(0)$ by antisymmetry property of $A_{1} \times A_{2}$.

$\Rightarrow x \wedge y=0$, since $\mathrm{f}$ is one- to- one

$\Rightarrow A_{1}(x \wedge y, 0)>0$, and $A_{2}(x \wedge y, 0)>0$.

Let $(a, b)$ be a maximal element of $\left(R_{1} \times R_{2}, A_{1} \times A_{2}\right)$.
Then $\quad\left(A_{1} \times A_{2}\right)(f(x \vee y),(a, b))=\left(A_{1} \times A_{2}\right)(f(x) \vee$ $f(y),(a, b))$ since $f$ is fuzzy lattice homomorphism.

$=\left(A_{1} \times A_{2}\right)((a, 0) \vee(0, b),(a, b))=\left(A_{1} \times A_{2}\right)((a \vee 0,0 \vee$ b), $(a, b))$

$=\min \left\{A_{1}(a \vee 0,0) \cdot A_{2}(0 \vee b, 0)\right\}=\min \left\{A_{1}(0,0), A_{2}(0,0)\right\}$

$=\min \{1,1\}=1>0$,

Hence $\left(A_{1} \times A_{1}\right)(f(x \vee y),(a, b))>0 . \quad$ Similarly, $\left(A_{1} \times A_{2}\right)((a, b), f(x \vee y))>0$. So that we have $f(x \vee y)=(a, b)$. Hence, $f(x \vee y)$ is maximal, since $(a, b)$ is maximal. So that we have $x \vee y$ is the maximal elements of $(R, A)$, since $\mathrm{f}$ is on to. Therefore, $x \in B_{A}(R)$.

Definition 33: Let $(R, A)$ be an ADFL. For $a, b \in R$ with $A(a, b)>0$ and $x \in[a, b]$. Then $y$ is a relative complement of $x$ in $[a, b]$ if and only if $A(x \wedge y, a)>0$ and $A(b, x \vee y)>0$, where $a$ is the least element and $b$ is the greatest element.

Definition 34: An ADFL $(R, A)$ is said to be relatively complemented ADFL if $([a, b], A)$ is a complemented fuzzy lattice for any $a, b \in R$ with $A(a, b)>0$.

Theorem 35: Let $(R, A)$ be an ADFL. Then $B_{A}(R)$ is a relatively complemented ADFL under induced operations of $(R, A)$.

Proof: Let $(R, A)$ be an ADFL. For $0 \in R$, there exist $a \in R$ such that $A(0 \wedge a, 0)=A(0,0)=1>0$ since $0 \wedge a=0$ in $\mathrm{R}$ and $A((a \vee 0) \vee a, a \vee 0)=A(a \vee 0, a \vee 0)=1>0$. since $a \vee 0$ is maximal. Hence $0 \in B_{A}(R)$, so that we have $B_{A}(R)$ is a non-empty subset of $(R, A)$.

Let $a_{1}, a_{2} \in B((R, A))$ and let $b_{1}, b_{2} \in(R, A)$ be a complement of $a_{1}$ and $a_{2}$ respectively. Then, $A\left(a_{1} \wedge b_{1}, 0\right)>0$, and $A\left(\left(a_{1} \vee b_{1}\right) \vee x, a_{1} \vee b_{1}\right)>0$ for $x \in R . A\left(a_{2} \wedge b_{2}, 0\right)>0$, and $A\left(\left(a_{2} \vee b_{2}\right) \vee y, a_{2} \vee b_{2}\right)>0$,for $y \in R$. Now, $A\left(\left(a_{1} \wedge a_{2}\right) \wedge\left(b_{1} \vee b_{2}\right), 0\right)$

$=A\left(\left[\left(a_{1} \wedge a_{2}\right) \wedge b_{1}\right] \vee\left[\left(a_{1} \wedge a_{2}\right) \wedge b_{2}\right], 0\right)$

$=A\left(\left[\left(a_{1} \wedge b_{1}\right) \wedge a_{2}\right] \vee\left[a_{1} \wedge\left(a_{2} \wedge b_{2}\right)\right], 0\right)$

since $\left(a_{1} \wedge a_{2}\right) \wedge b_{1}=\left(a_{1} \wedge b_{1}\right) \wedge a_{2}$, and

$\left(a_{1} \wedge a_{2}\right) \wedge b_{2}=a_{1} \wedge\left(a_{2} \wedge b_{2}\right)$

$=A\left(\left(0 \wedge a_{2}\right) \vee\left(a_{1} \wedge 0\right), 0\right)$, since $a_{1} \wedge b_{1}=0$, and $a_{2} \wedge b_{2}=0$.

$=A(0,0)=1>0$.

Hence $A\left(\left(a_{1} \wedge a_{2}\right) \wedge\left(b_{1} \vee b_{2}\right), 0\right)>0 . \quad$ Similarly, $A\left(0,\left(a_{1} \wedge a_{2}\right) \wedge\left(b_{1} \vee b_{2}\right)\right)>0$. We get $\left(a_{1} \wedge a_{2}\right) \wedge\left(b_{1} \vee b_{2}\right)=0$ by antisymmetry property of A. Again for any $x \in R$, then $A\left(\left[\left(a_{1} \wedge a_{2}\right) \vee\left(b_{1} \vee b_{2}\right)\right] \wedge x, x\right)$

$=A\left(\left[\left(\left(a_{1} \wedge a_{2}\right) \vee b_{1}\right) \vee b_{2}\right] \wedge x, x\right)$

$=A\left(\left[\left(\left(a_{1} \vee b_{1}\right) \wedge\left(a_{2} \vee b_{1}\right) \vee b_{2}\right] \wedge x, x\right)\right.$ by Theorem 10

$=A\left(\left[\left(a_{2} \vee b_{1}\right) \vee b_{2}\right] \wedge x, x\right)$, since $a_{1} \vee b_{1}$ is maximal

$=A\left(\left[\left(a_{2} \vee b_{1}\right) \vee b_{2}\right] \wedge x, x\right)$

$=A\left(\left(b_{2} \vee a_{2}\right) \wedge x, x\right)$, since $a_{2} \vee b_{2}$ is maximal. We have $b_{2} \vee a_{2}$ is also maximal.

$=A(x, x)=1>0$.

Hence $A\left(\left[\left(a_{1} \wedge a_{2}\right) \vee\left(b_{1} \vee b_{2}\right)\right] \wedge x, x\right)>0$. Similarly, $A\left(x,\left[\left(a_{1} \wedge a_{2}\right) \vee\left(b_{1} \vee b_{2}\right)\right] \wedge x\right)>0$. So that we have $\left.\left[\left(a_{1} \wedge a_{2}\right) \vee\left(b_{1} \vee b_{2}\right)\right] \wedge x\right)=x$ and hence $\left(a_{1} \wedge a_{2}\right) \vee\left(b_{1} \vee b_{2}\right)$ is maximal. Therefore, $a_{1} \wedge a_{2} \in B_{A}(R)$. To show $a_{1} \vee a_{2} \in B_{A}(R)$. Let $(R, A)$ be an ADFL. Then $A\left(\left(b_{1} \wedge b_{2}\right) \wedge\left(a_{1} \vee a_{2}\right), 0\right)$

$=A\left(\left[\left(b_{1} \wedge b_{2}\right) \wedge a_{1}\right] \vee\left[\left(b_{1} \wedge b_{2}\right) \wedge a_{2}\right], o\right)$

$\left.=A\left(\left(b_{1} \wedge a_{1}\right) \wedge b_{2} \vee\left(b_{1} \wedge b_{2}\right) \wedge a_{2}\right), 0\right)$

$=A\left(\left(b_{1} \wedge a_{1} \wedge b_{2}\right) \vee\left(b_{1} \wedge\left(b_{2} \wedge a_{2}\right), 0\right)\right.$, since $b_{1} \wedge a_{1}=0$ and $b_{2} \wedge a_{2}=0$

$=A\left(\left(0 \wedge b_{2}\right) \vee\left(b_{1} \wedge 0\right), 0\right)=A(0 \vee 0,0)=A(0,0)=1>0$ 
We get $A\left(\left(b_{1} \wedge b_{2}\right) \wedge\left(a_{1} \vee a_{2}\right), 0\right)>0$. Similarly, $A\left(0,\left(b_{1} \wedge b_{2}\right) \wedge\left(a_{1} \vee a_{2}\right)\right)>0$. Hence $\left(b_{1} \wedge b_{2}\right) \wedge\left(a_{1} \vee a_{2}\right)=0$ by antisymmetry property of A. For any $y \in$ $R, A\left(\left[\left(b_{1} \wedge b_{2}\right) \vee\left(a_{1} \vee a_{2}\right)\right] \wedge y, y\right)$

$=A\left(\left[\left(a_{1} \vee a_{2}\right) \vee\left(b_{1} \wedge b_{2}\right)\right] \wedge y, y\right)$ by theorem 10 .

$=A\left(\left[\left(\left(a_{1} \vee a_{2}\right) \vee b_{1}\right) \wedge\left(\left(a_{1} \vee a_{2}\right) \vee b_{2}\right)\right] \wedge y, y\right) L D \vee$ and theorem 10.

$=A\left(\left[\left(\left(b_{1} \vee a_{1}\right) \vee a_{2}\right) \wedge\left(a_{1} \vee\left(a_{2} \vee b_{2}\right)\right)\right] \wedge y, y\right)$

$=A\left(\left[\left(b_{1} \vee a_{1}\right) \wedge\left(a_{2} \vee b_{2}\right] \wedge y, y\right), \quad\right.$ since $b_{1} \vee a_{1}, a_{2} \vee b_{2}$ are maximals.

$=A(y, y)=1>0$.

Hence $A\left(\left[\left(b_{1} \wedge b_{2}\right) \vee\left(a_{1} \vee a_{2}\right)\right] \wedge y, y\right)>0$. Similarly, $A\left(y,\left[\left(b_{1} \wedge b_{2}\right) \vee\left(a_{1} \vee a_{2}\right)\right] \wedge y\right)>0 . \quad$ Therefore, $\left[\left(b_{1} \wedge b_{2}\right) \vee\left(a_{1} \vee a_{2}\right)\right] \wedge y=y$. Thus, $\left(b_{1} \wedge b_{2}\right) \vee\left(a_{1} \vee a_{2}\right)$ is maximal. So that $b_{1} \wedge b_{2}$ is a complement of $a_{1} \vee a_{2}$ and $a_{1} \vee a_{2} \in B(L)$. Therefore $B_{A}(R)$ is an ADFL under induced operations of $(R, A)$. Let $(R, A)$ be an ADFL, and let $a, b \in B_{A}(R)$, then there exist $c, d \in R$ such that $A(a \wedge c, 0)>0$, and $A(b \wedge d, 0)>0$, and $A((a \vee c) \vee x, a \vee c)>0$ and $A((b \vee d) \vee x, b \vee d)>0$, for all $x \in R$ as $a \vee c, b \vee d$ are maximals. Now, $A((c \wedge b) \wedge(a \vee d), 0)$

$=A(((c \wedge b) \wedge a) \vee((c \wedge b) \wedge d), 0)$

$=A((c \wedge a \wedge b) \vee(c \wedge b \wedge d), 0), \quad \wedge \quad$ is associative,

$a \wedge c=c \wedge a=0$ and $b \wedge d=0$

$=A((0 \wedge b) \vee(c \wedge 0), 0)$

$=A(0,0)=1>0$.

Hence $A((c \wedge b) \wedge(a \vee d), 0)>0 . \quad$ Similarly, $A(0,(c \wedge b) \wedge(a \vee d))>0$. Therefore, $(c \wedge b) \wedge(a \vee d)=0$ by antisymmetry property of $\mathrm{A}$. For any $y \in R$, and $(R, A)$ be an ADFL. $A([(c \wedge b) \vee(a \vee d)] \wedge y, y)$

$=A([(a \vee d) \vee(c \wedge b)] \wedge y, y)$

$=A([((a \vee d) \vee c) \wedge((a \vee d) \vee b)] \wedge y, y)$

$=A([((c \vee a) \vee d) \wedge((a \vee(d \vee b)] \wedge y, y)$, since $\wedge$ is associative by Theorem 6 .

$=A([(c \vee a) \wedge(d \vee b))] \wedge y, y)$

$=A(y, y)=1>0$, since $a \vee c$, and $b \vee d$ are maximal.

Hence $A([(c \wedge b) \vee(a \vee d)] \wedge y, y)>0$. Similarly, $A(y,[(c \wedge b) \vee(a \vee d)] \wedge y)>0$. Therefore, $[(c \wedge b) \vee(a \vee d)] \wedge y=y$ by antisymmetry property of A. $(c \wedge b) \vee(a \vee d)$ is maximal. Hence $c \wedge b \in B_{A}(R)$. Now, $A(a \wedge(c \wedge b), 0)=A((a \wedge c) \wedge b, 0)$, since $\wedge$ is associative:

$=A(0 \wedge b, 0)=A(0,0)=1>0$

We have $A(a \wedge(c \wedge b), 0)>0$. Similarly, $A(0, a \wedge(c \wedge b))>0$. Therefore, $a \wedge(c \wedge b)=0$ by antisymmetry property of $\mathrm{A}$. $A(a \vee(c \wedge b), a \vee b)=A((a \vee c) \wedge(a \vee b), a \vee b)$

$=A(a \vee b, a \vee b)=1>0$

Hence $A(a \vee(c \wedge b), a \vee b)>0$. Similarly, $A(a \vee b, a \vee(c \wedge b))>$ 0 . Therefore, $a \vee(c \wedge b)=a \vee b$ by antisymmetry property of A. Therefore, $B((R, A))$ is relatively complemented ADFL.

Theorem 36: Let $(R, A)$ be an ADFL.Then $B(R)$ is a Birkhoff center of an ADL R if and only if $B_{A}(R)$ is a Birkhoff center of an $\operatorname{ADFL}(R, A)$.

Proof: Assume $B(R)$ be a Birkhoff center $R$ and let $a \in$ $B(R)$. Then, there exist $b \in R$ such that $a \wedge b=0$, and $a \vee b$ is maximal. Let $(R, A)$ be an ADFL. $A(a \wedge b, 0)=A(a \wedge(0 \vee b), 0)$ $=A((a \wedge 0) \vee(a \wedge b), 0)$, since $a \wedge b=0$

$=A(0 \vee 0,0)=A(0,0)=1$.

$\Rightarrow A(a \wedge b, 0)>0$
$A(a \vee b) \vee x, a \vee b)=A((a \vee b) \vee((a \vee b) \wedge x), a \vee b)$

$=A(a \vee b, a \vee b)=1>0$ since $a \vee b$ is maximal.

We get $A(a \wedge b, 0)>0$ and $A((a \vee b) \vee x, a \vee b)>$ 0 ,for all $x \in R$.

Therefore, $B_{A}(R)$ is a Birkhoff center of an ADFL $(R, A)$. Conversely, assume for $a \in B_{A}(R)$. Then there exist $b \in R$ such that $A(a \wedge b, 0)>0$ and $A((a \vee b) \vee x, a \vee b)>0$, for all $x \in R$. Since 0 is least element, we have $0 \leq a \wedge b, a, b \in R$ $\Rightarrow A(a \wedge b, 0)>0$ and $A(0, a \wedge b)>0$

$\Rightarrow a \wedge b=0$ by antisymmetry property of $A$, and $a \leq(a \vee b) \vee$ $x, b \leq(a \vee b) \vee x \Rightarrow a \vee b \leq(a \vee b) \vee x$. Hence $A(a \vee b,(a \vee b) \vee$ $x)>0$. We have $(a \vee b) \vee x=a \vee b$. Therefore, $a \vee b$ is maximal. Hence $a \in B(R)$.

Theorem 37: Let $(R, A)$ be an ADFL.Then $(R, A)$ is relatively complemented if and only if $B_{A}(R)=(R, A)$.

Proof: Suppose $(R, A)$ is a relatively complemented ADFL. Let $x \in R$ such that $A(x \vee m, m)=1$. Then $m \vee x=m$ is maximal in $(R, A)$ implies that $A(x, m)>0$. Since $(R, A)$ is relatively complemented, there exists $y \in R$ such that $A(x \wedge y, 0)=A(0, x \wedge y)=1$ and $A((x \vee y) \vee m, x \vee y)>0$, and $x \leq(x \vee y) \vee m, y \leq(x \vee y) \vee m$

$\Rightarrow x \vee y \leq(x \vee y) \vee m$.

Hence $A(x \vee y,(x \vee y) \vee m)>0$. We get $(x \vee y) \vee m=x \vee y$ by antisymmetry property of $A$. So that $x \vee y$ is maximal. Therefore, $x \in B_{A}(R)$ implies that $(R, A) \subseteq B_{A}(R)$. Clearly $B_{A}(R) \subseteq(R, A)$. Thus, $B_{A}(R)=(R, A)$. The converse follows by Theorem 35 .

Theorem 38: Let $\left(R_{1}, A\right)$ and $\left(R_{2}, A\right)$ be $A D F L_{s}$. Then $B_{A_{1} \times A_{2}}\left(R_{1} \times R_{2}\right)=B_{A_{1}}\left(R_{1}\right) \times B_{A_{2}}\left(R_{2}\right)$.

Proof: Let $\left.\left(R_{1} \times R_{2}, A_{1} \times A_{2}\right)\right)$ be an ADFL and $(a, c) \in B_{A_{1} \times A_{2}}\left(R_{1} \times R_{2}\right)$. Then there exist $(b, d) \in R_{1} \times R_{2}$ such that $\left(A_{1} \times A_{2}\right)((a, c) \wedge(b, d),(0,0))>0$, by definition of Birkhoff center in ADFL.

$\Leftrightarrow\left(A_{1} \times A_{2}\right)((a \wedge b, c \wedge d),(0,0))>0$

Hence $\left(A_{1} \times A_{2}\right)((a \wedge b, c \wedge d),(0,0))>0$. Similarly, $\left(A_{1} \times A_{2}\right)((0,0),(a \wedge b, c \wedge d))>0$. We have $(a \wedge b, c \wedge d)=(0,0)$.

$\Rightarrow a \wedge b=0$ and $c \wedge d=0$

$\Rightarrow A_{1}(a \wedge b, 0)>0 \quad, \quad A_{2}(c \wedge d, 0)>0$ and $\left(A_{1} \times A_{2}\right)(((a, c) \vee(b, d)) \vee(x, y),(a, c) \vee(b, d))>0$, for $(x, y) \in R_{1} \times R_{2}$.

$\Leftrightarrow\left(A_{1} \times A_{2}\right)(((a \vee b) \vee x,(c \vee d) \vee y),(a \vee b, c \vee d))>0$ for $(x, y) \in R_{1} \times R_{2}$.

Hence $\left(A 1 \times A_{2}\right)(((a \vee b) \vee x,(c \vee d) \vee y),(a \vee b, c \vee d))>0$. Similarly, $\left(A_{1} \times A_{2}\right)((a \vee b, c \vee d),((a \vee b) \vee x,(c \vee d) \vee y)>0$ implies that $((a \vee b) \vee x,(c \vee d) \vee y)=(a \vee b, c \vee d)$.

$\Rightarrow(a \vee b) \vee x=a \vee b$ and $(c \vee d) \vee y=c \vee d$.

So that we get $a \vee b$ is maximal in $\left(R_{1}, A_{1}\right)$ and $c \vee d$ is maximal in $\left(R_{2}, A_{2}\right)$.

$\Rightarrow a \in B_{A_{1}}\left(R_{1}\right)$ and $c \in B_{A_{2}}\left(R_{2}\right)$.

$\Rightarrow(a, c) \in B_{A_{1}}\left(R_{1}\right) \times B_{A_{2}}\left(R_{2}\right)$. Therefore, $B_{A_{1} \times A_{2}}\left(R_{1} \times R_{2}\right) \subseteq$ $B_{A_{1}}\left(R_{1}\right) \times B_{A_{2}}\left(R_{2}\right)$.

Conversely, suppose $(a, c) \in B_{A_{1}}\left(R_{1}\right) \times B_{A_{2}}\left(R_{2}\right)$. It implies that $a \in B_{A_{1}}\left(R_{1}\right)$ and $c \in B_{A_{2}}\left(R_{2}\right)$, then there exist $b \in R_{1}$ and $d \in R_{2}$ such that $A_{1}(a \wedge b, 0)>0$ and $A_{2}(c \wedge d, 0)>0$. Again $A_{1}((a \vee b) \vee x, a \vee b)>0$ and $A_{2}((c \vee d) \vee y, c \vee d)>0$. Since $a \leq(a \vee b) \vee x, b \leq(a \vee b) \vee x$.

$\Rightarrow a \vee b \leq(a \vee b) \vee x$. 
Hence $A_{1}(a \vee b,(a \vee b) \vee x)>0$. So that we get $(a \vee b) \vee x=$ $a \vee b$. This implies that $a \vee b$ is the maximal element in $\left(R_{1}, A_{1}\right)$.

$c \leq(c \vee d) \vee y, d \leq(c \vee d) \vee y$.

$\Rightarrow c \vee d \leq(c \vee d) \vee y$.

Hence $A_{2}(c \vee d,(c \vee d) \vee y)>0$, which implies that $(c \vee d) \vee y=$ $c \vee d$, for all $y \in R_{2}$ by antisymmetry property of $A_{2}$.

$c \vee d$ is a maximal element in $\left(R_{2}, A_{2}\right)$. It implies that $(a, c) \in$ $B_{A_{1} \times A_{2}}\left(R_{1} \times R_{2}\right)$. Hence $B_{A_{1}}\left(R_{1}\right) \times B_{A_{2}}\left(R_{2}\right) \subseteq B_{A_{1} \times A_{2}}\left(R_{1} \times\right.$ $\left.R_{2}\right)$. Therefore, $B_{A_{1} \times A_{2}}\left(R_{1} \times R_{2}\right)=B_{A_{1}}\left(R_{1}\right) \times B_{A_{2}}\left(R_{2}\right)$.

\section{REFERENCES}

[1] U. Swamy and G. Rao, "Almost distributive lattices," Journal of the Australian Mathematical Society (Series A), vol. 31, no. 1, pp. 77-91, 1981.

[2] U. Swamy, G. Rao, R. Kumar, and C. Pragati, "Birkhoff centre of a poset," Southeast Asian Bulletin of Mathematics, vol. 26, no. 3, pp. 509-516, 2003.

[3] U. Swamy and S. Ramesh, "Birkhoff centre of an almost distributive lattice," International Journal of Algebra, vol. 3, no. 11, pp. 539-546, 2009.

[4] L. Zadeh, "Fuzzy sets," Information and control, vol. 8, no. 3, pp. 338 $353,1965$.

[5] J. Goguen, "L-fuzzy sets," Journal of mathematical analysis and applications, vol. 18, no. 1, pp. 145-174, 1967.

[6] E. Sanchez, "Resolution of composite fuzzy relation equations," Information and control, vol. 30, no. 1, pp. 38-48, 1976

[7] Y. Bo and W. Wangming, "Fuzzy ideals on a distributive lattice," Fuzzy sets and systems, vol. 35, no. 2, pp. 231-240, 1990.

[8] G. Rao, "Almost distributive lattice," Ph.D. dissertation, Department of Mathematics, Andhra University, Visakhapatnam, 1980.

[9] I. Chon, "Fuzzy partial order relations and fuzzy lattices," Korean $J$. Math, vol. 17, no. 4, pp. 361-374, 2009.

[10] A. Berhanu, G. Yohanes, and T. Bekalu, "Almost distributive fuzzy lattice," To be communicated. 